\title{
D-dimer as a predictive marker for COVID-19 severity
}

\author{
Rim Harfouch ${ }^{1}$ \\ ${ }^{1}$ Affiliation not available
}

February 2, 2022

\section{Rim M. Harfouch}

Department of microbiology and biochemistry, Faculty of pharmacy, Al Sham private university, Latakia, Syria.

Correspondence Author's E-mail: r.h.foph.lat@aspu.edu.sy

\begin{abstract}
The 2019 coronavirus disease (COVID-19) presents with a large variety of clinical manifestations ranging from asymptomatic carrier state to severe respiratory distress, multiple organ dysfunction and death. While it was initially considered primarily a respiratory illness, rapidly accumulating data suggests that COVID19 results in a unique, profoundly prothrombotic milieu leading to both arterial and venous thrombosis. Consistently, elevated D-dimer level has emerged as an independent risk factor for poor outcomes, including death. The pathophysiology underlying the hyper-coagulation state is poorly understood. However, a growing body of data suggests that the initial events occur in the lung. A severe inflammatory response, originating in the alveoli, triggers a dysfunctional cascade of inflammatory thrombosis in the pulmonary vasculature, leading to a state of local coagulopathy. This is followed by a generalized hyper-coagulation state that results in and microvascular thrombosis. Of concern, is the observation that anticoagulation may be inadequate in many circumstances, highlighting the need for alternative or additional therapies. Numerous ongoing studies investigating the pathophysiology of the COVID-19 associated coagulopathy may provide mechanistic insights that can direct appropriate interventional strategies.
\end{abstract}

\section{Keywords:}

COVID-19, D-dimer, Hyper-coagulation, Predictive Marker.

\section{Introduction}

Severe acute respiratory syndrome coronavirus 2 (SARS COV2) caused Coronavirus disease 2019 (COVID19), which was first recorded in Wuhan, China in December 2019 [1]. COVID-19 is primarily a respiratory infection, but it can also affect multiple organ systems including cardiac, neurological, gastrointestinal and renal systems [2]. Viremia and cytokine storm are common in COVID-19 especially in immune-compromised patients, and that may activate the coagulation cascade which causes thrombotic complications and coagulopathies including cardiac thrombosis and disseminated intravascular coagulopathy [3].

D-dimer is a fibrin degradation product, which is used as a biomarker for thrombotic disorders. It is considered normal with values less than $0.5 \mu \mathrm{g} / \mathrm{mL}$, and the level of D-dimer rises in severe communityacquired pneumonia, it is also used as an initial screening test to diagnose patients who have signs, or symptoms suggestive of venous thromboembolism [4]. D-dimer is detectable in patients with deep venous thrombosis (DVT), as it is a marker of endogenous fibrinolysis. Following the outbreak of the COVID-19 pandemic, D-dimer has been identified as a potential indicator for its prognosis in COVID-19 patients. [5]. 


\section{Cytokine storm in COVID-19}

Cytokine release plays a vital role in the immune-pathology against a virus, but over-expressions of immune mediators may cause severe damage to the human organ systems. Patients suffering from COVID-19 have been diagnosed with overexpression of monocyte chemo-attractant protein (MCP-1), IFN- $\gamma$, IP-10, and IL13. These pro-inflammatory cytokines may cause activation of responses from the T-helper type 1 cells (Th1). COVID-19 patients have also shown high levels of cytokines secreted by Th2 cells like IL-10 and IL-4.

These two cytokines are involved in inhibition of the inflammatory response. Furthermore, the levels of IL-6 and IL-2 R, obtained from serum, depict a positive correlation with the disease severity, which means they are highest in patients who are critically ill and lowest in ordinary patients. Another study on patients in Wuhan, China, compared the COVID-19 patients from the general wards and the intensive care units (ICUs). The latter were reported to exhibit increased levels of serum IP-10, macrophage inflammatory protein-1A, granulocyte colony-stimulating factor and TNF- $\alpha$. The above studies act as evidence of the positive correlation between the 'cytokine storm' and COVID-19 progression [6].

The mechanism of cytokine storm syndrome depends on the imbalance of pro-inflammatory and antiinflammatory process and the interaction of specific cells and cytokines, resulting in immune regulation disorder. Cytokines can be markedly increased in patients with cytokine storm syndrome, which differ according to the heterogeneity of the disease. A previous study found that H5N1 infected patients had higher levels of interferon-gamma-induced protein-10 (IP-10), monocyte chemo-attractant protein 1 (MCP-1) and IL-8 than patients with seasonal H1N1 influenza. Moreover, it was confirmed that cytokines play an important role in the pathogenesis of severe $\mathrm{CoV}$ infection. Spleen and lymph node atrophy are observed in patients with COVID-19, while lymphadenopathy and splenomegaly are more common in other cytokine storm syndrome related diseases [7].

\section{D-dimer and COVID-19}

Thromboembolism of COVID-19 patients is the fatal sequelae of hyper-coagulation and fibrinolytic abnormalities. Pulmonary embolism and deep vein thrombosis (DVT) can cause respiratory failure in severely ill patients with COVID-19. After death pathology shows that small fibrinous thrombi in small pulmonary arterioles are very common. Activation of the coagulation cascade is further supported by endothelial tumefaction, pulmonary megakaryocytes in the capillaries, and endotheliitis. Elevated D-dimer is an indicator of the activation of the fibrinolysis system and removal of clots or extravascular collections of fibrin by plasmin $[8]$.

Increased D-dimer level has not consistently been observed by all COVID-19 clinical studies, although it is a broadly applied biomarker for prognosis and outcomes of anti-thrombosis. There is strong positive association of elevated D-dimer on admission with mortality, indicating the prognostic value of an elevated D-dimer for the high risk of death. There is positive correlation between D-dimer and days from onset to admission, the need for ventilation and the days are taken for PCR test reversion to negative. Data shows that prompt admission and clearance of the SARS-CoV-2 virus may alleviate the severity and reduce fatal events by preventing hyper-fibrinolysis and inflammation.

D-dimer and other clinical variables' associations indicate either a relationship that could be cause-effect or indirect. Few D-dimer-associated variables have been confirmed as prognostic biomarkers for developing fatal events and in-hospital mortality. Extremely elevated plasma D-dimer seems to be the consequence of hyper-fibrinolysis predominately in the pulmonary capillaries and other organs. And a dynamic increase in the D-dimer level may be associated with thromboembolism and higher fatality, while we infer that a continuous decline by daily testing will generally lead to recovery. D -dimer was positively associated with the severity of lung injury, the days from the onset to admission, onset to dyspnea, time is taken to be PCR negative, and overall mortality. Finally, the variables were significantly associated with D-dimer were respiratory rate, systolic pressure, dyspnea, serum $\mathrm{K}^{+}$, neutrophils, globulin, CRP, ferritin, blood glucose, total bilirubin, mortality, ventilation, sepsis, and acute cardiac injury [9]. 
The coagulopathy and abnormal results in coagulation tests have become common features reported in patients with COVID-19 from the very early days of the emergence of the new coronavirus strain. COVID-19dependent coagulopathy gained attention when PT, aPTT, fibrinogen, and D-dimer tests were recommended by researchers to evaluate the proper homeostasis of the system associated with the prognosis of patients. The prophylactic use of anticoagulants was proven to be effective in lowering the mortality rate and highlighted the role of the coagulation system in COVID-19. The link between thrombosis and COVID-19 as an inflammatory disease has been investigated [10].

\section{Conclusion:}

The alterations in the hemostatic balance in COVID-19 patients are strongly disturbed and contribute to a high prothrombotic status. The high rate of venous thromboembolism in COVID-19 patients goes along with derangements in coagulation laboratory parameters.

Hemostasis testing has an important role in diagnosed COVID-19 patients. Elevated D-dimer levels were found to be a crucial laboratory marker in the risk assessment of thrombosis in COVID-19 patients.

\section{References:}

1. Harfouch RM, Moualla YM, Shahoud SM, Bela l LA, Mohammad BS, Mustafa NM, et al., Epidemiology of COVID-19 in the most pandemic Countries: A review article. Acad .J. Biotechnol. 2021;9:21-7.

2. Harfouch RM, Alshaikh S, Alshimaly M, Assaad A, Ahmad J, Zoughaibi H, et al., Therapeutic approaches for COVID 19: Challenges and successes. Ann Clin Anal Med. 2020.

3. Savla SR, Prabhavalkar KS, Bhatt LK. Cytokine storm associated coagulation complications in COVID-19 patients: Pathogenesis and Management. Expert Rev Anti Infect Ther . 2021;19(11):13971413. doi:10.1080/14787210.2021.1915129

4. Poudel A, Poudel Y, Adhikari A, Aryal BB, Dangol D, Bajracharya T, Maharjan A, Gautam R. Ddimer as a biomarker for assessment of COVID-19 prognosis: D-dimer levels on admission and its role in predicting disease outcome in hospitalized patients with COVID-19. PLoS One. 2021 Aug 26;16(8):e0256744. doi: 10.1371/journal.pone.0256744. PMID: 34437642; PMCID: PMC8389366.

5. Zhao R, Su Z, Komissarov AA, et al. Associations of D-Dimer on Admission and Clinical Features of COVID-19 Patients: A Systematic Review, Meta-Analysis, and Meta-Regression. Front Immunol . 2021;12:691249. Published 2021 May 7. doi:10.3389/fimmu.2021.691249

6. Savla SR, Prabhavalkar KS, Bhatt LK. Cytokine storm associated coagulation complications in COVID-19 patients: Pathogenesis and Management. Expert Rev Anti Infect Ther . 2021;19(11):13971413. doi:10.1080/14787210.2021.1915129

7. Harfouch RM. Cytokine Storm Syndrome in COVID-19 Patients: Characteristics and Diagnosis. Ann Clin Med Case Rep. 2021;7(18):1-3.

8. Abou-Ismail MY, Diamond A, Kapoor S, Arafah Y, Nayak L. The hypercoagulable state in COVID-19: Incidence, pathophysiology, and management [published correction appears in Thromb Res. 2020 Nov 26;:]. Thromb Res . 2020;194:101-115. doi:10.1016/j.thromres.2020.06.029

9. Zhao R, Su Z, Komissarov AA, et al. Associations of D-Dimer on Admission and Clinical Features of COVID-19 Patients: A Systematic Review, Meta-Analysis, and Meta-Regression. Front Immunol . 2021;12:691249. Published 2021 May 7. doi:10.3389/fimmu.2021.691249.

10. Connors JM, Levy JH. COVID-19 and its implications for thrombosis and anticoagulation. Blood . 2020;135(23):2033-2040. doi:10.1182/blood.2020006000. 